\title{
ANÁLISIS DE ALGUNOS CRITERIOS PARA DIFERENCIAR ENTRE CIENCIA Y TECNOLOGÍA
}

ACEVEDO DÍAZ, JOSÉ ANTONIO

Servicio de Inspección. Delegación Provincial de Educación y Ciencia. Alameda Sundheim, 17. Huelva.

\begin{abstract}
SUMMARY
Up till now, the STS education has not managed to discern rightly between science and technology. In the paper, this status is briefly described, and we analyze three criteria to clarify the confusing panorama existing in this domain: the specific characteristics of the technological knowledge; the attitudes towards publications; and the purposes of the academic and industrial research laboratories.
\end{abstract}

\section{BREVE PANORAMA DE UNA CONFUSIÓN}

Según Fleming (1987), la ciencia y la tecnología, como consecuencia de una interdependencia mal entendida entre ambas, suelen identificarse como una empresa única capaz de afectar a la sociedad: la tecnociencia, que aboga por alcanzar una comprensión de las diferencias existentes entre ambas.

La gran mayoría de los intentos realizados para introducir algunos conocimientos de tecnología en la enseñanza de las ciencias, desde la perspectiva de ciencia integrada con tecnología (Allsop y Woolnough, 1990; Casalderrey, 1986; Fernández-Uría, 1979; UNESCO, 1990; Woolnough, 1988), ha contribuido más bien a reforzar una visión deformada de la tecnología jerárquicamente subordinada a la ciencia o a favorecer su identificación errónea con la ciencia aplicada (Acevedo, 1995, 1996 c). Esta imagen, muy arraigada popularmente, se ha ido extendiendo desde la ciencia a través de la divulgación científica y de la didáctica de las ciencias experimentales, como mínimo.

Así mismo, los esfuerzos que se vienen haciendo, desde la década de los años ochenta, para dar una orientación
CTS (ciencia, tecnología y sociedad) a los contenidos de los currículos de ciencia y tecnología no han contribuido, en general, a aclarar con cierto rigor las relaciones y diferencias entre ciencia y tecnología, a pesar de que entre sus objetivos se encuentra la mejor comprensión de la naturaleza de éstas. Incluso, en muchos casos, se ha conseguido favorecer la confusión en este terreno. Por ejemplo, en las primeras versiones del SATIS (Science and Technology in Society), conocido conjunto de materiales curriculares CTS de Gran Bretaña, la tecnología se define como «el proceso por medio del cual se hace posible la aplicación de la ciencia para satisfacer las necesidades humanas», lo que, sin duda, supone dar un punto de vista sesgado.

También han favorecido el desconcierto, a la hora de distinguir entre ciencia y tecnología, algunos historiadores sociales de la ciencia, como, por ejemplo, Bernal (1964) cuando dice que:

«La principal ocupación del científico es encontrar el modo de hacer las cosas, mientras que la del ingeniero consiste en hacerlas.» (p. 42 de la traducción española). 
Tampoco ayudan demasiado a aclarar este panorama algunas de las orientaciones oficiales para los nuevos currículos españoles de la educación secundaria obligatoria, tal y como se desprende de la lectura de los dos siguientes párrafos extraídos de las mismas.

«A lo largo de este último siglo, las ciencias de la naturaleza han ido incorporándose progresivamente a la sociedad y a la vida social, convirtiéndose en una de las claves esenciales para entender la cultura contemporánea, por sus contribuciones a la satisfacción de necesidades humanas. Por eso mismo, la sociedad ha tomado conciencia de la importancia de las ciencias y de su influencia en asuntos como la salud, los recursos alimenticios y energéticos, la conservación del medio ambiente, el transporte y los medios de comunicación.» (De la introducción al área de Ciencias de la Naturaleza que aparece en el RD 1007/91, anexo I, p. 35, MEC, 1991).

«La ciencia y la tecnología tienen propósitos diferentes: la primera trata de ampliar y profundizar el conocimiento de la realidad; la segunda, de proporcionar medios y procedimientos para satisfacer necesidades. Pero ambas son interdependientes y se potencian mutuamente. Los conocimientos de la ciencia se aplican en desarrollos tecnológicos; determinados objetos o sistemas creados por aplicación de la tecnología son imprescindibles para avanzar en el trabajo científico; las nuevas necesidades que surgen al tratar de realizar los programas de investigación científica plantean retos renovados a la tecnología. Comprender estas relaciones entre ciencia y tecnología constituye un objetivo educativo de la etapa.» (De la introducción al área de Tecnología que aparece en el RD 1007/1991, anexo I, p. 74, MEC, 1991).

El primero de ellos podría estar refiriéndose con toda propiedad más a la tecnología que a la propia ciencia, de acuerdo con lo que se indica explícitamente en el segundo texto. En cualquier caso, transmite una visión «utilitarista» de ésta casi exclusivamente centrada en su dimensión tecnológica, una perspectiva que es coherente con el nuevo paradigma de desarrollo e investigación (D+I) -en ese orden- que está empezando a dominar la política científica y tecnológica de finales de este siglo (Acevedo, 1997).

Esto contrasta con lo que se dice en el segundo párrafo, donde se destaca sobre todo el punto de vista «teoricista» e «idealista» de la ciencia académica. En éste parece que la diferencia entre la ciencia y la tecnología se establece atribuyendo a la primera la búsqueda de conocimiento y comprensión sobre el mundo natural, y la de soluciones a problemas prácticos de la vida cotidiana y de la propia ciencia a la segunda de ellas. Por otro lado, dejando aparte los aspectos epistemológicos relacionados con la naturaleza de la ciencia y de la tecnología, en el segundo texto subyace, en su conjunto, una visión jerarquizada de las relaciones entre ciencia y tecnología, en la que ésta se subordina a aquélla como instrumento útil para resolver sus necesidades. La tecnología se percibe de un modo servil hacia la ciencia para que ésta pueda seguir elaborando conocimiento teórico, que a su vez nutre a la tecnología; un punto de vista popular al que ha contribuido interesadamente la ciencia, pero profundamente deformado tal y como hemos subrayado más arriba.

Podrían multiplicarse ejemplos como los señalados (Layton, 1988; Price y Cross, 1995), pero creemos que los expuestos aquí son suficientes para ilustrar el confuso panorama existente en este terreno.

\section{CIENCIA, TECNOLOGÍA Y FINALIDADES EDUCATIVAS}

Las concepciones que se tengan de la ciencia y la tecnología, así como de sus relaciones y diferencias, condicionan en buena medida las finalidades y los objetivos de la educación científica y la educación tecnológica, lo que, a su vez, conduce a dotar de distintos significados a las expresiones alfabetización científica y alfabetización tecnológica, presentes ambas durante los últimos años en numerosos informes de política educativa de diversos países, sobre todo del ámbito cultural occidental.

Por ejemplo, en el caso de la tecnología Gilbert (1995) ha hecho notar que, puesto que no hay una definición de la misma que sea aceptada por la mayoría, aún menos la habrá para la educación tecnológica. La consecuencia obvia de esto será la formulación de distintas finalidades y objetivos para la enseñanza de la tecnología, y los consiguientes significados de alfabetización tecnológica (Acevedo, 1996c), según el punto de vista adoptado. De esta manera, puede considerarse necesaria la educación tecnológica para la orientación vocacional y la preparación preprofesional de la juventud ante el mundo laboral, para dar a conocer uno de los logros más importantes alcanzados por la humanidad en todos los tiempos, a fin de desarrollar capacidades para la evaluación y el uso responsable de la tecnología, etc., de acuerdo con las diferentes razones -principalmente económicas, culturales y sociales- que se suelen esgrimir con el fin de justificar la introducción de la tecnología en el currículo de la educación obligatoria (Gilbert, 1992; Medway, 1989).

Por otra parte, esta vez con referencia a la enseñanza de la ciencia, Reid y Hodson (1989) han destacado la importancia del principio de comprensividad denominado ciencia para todos en la educación secundaria obligatoria, que fue asumido hace aproximadamente veinte años por la política educativa del Reino Unido de Gran Bretaña y se ha venido potenciando desde entonces, tal y como se desprende de los siguientes párrafos extraídos de sendos documentos del Department of Education and Science (DES, 1977, 1985):

«La educación científica debe ser para todos -no solamente para aquéllos que tienen posibilidad de convertirse en científicos, tecnólogos o técnicos-. [...] Un curso de ciencias debe ser, por tanto, un componente esencial del currículo de cada alumno y alumna hasta el final de la escolarización obligatoria.» (DES, 1977). 
«La ciencia debería tener un lugar en la educación de todo el alumnado en edad de escolarización obligatoria, tanto si tienen posibilidades de seguir una carrera científica o técnica como si no. Se tendrían que introducir las ciencias para todos en la escuela primaria y continuar con un programa más amplio, perfectamente adecuado a sus capacidades y aptitudes, durante los cinco primeros años de la educación secundaria.» (DES, 1985).

Sin embargo, aunque tal principio parece estar recibiendo el apoyo de casi todo el mundo, necesita de una clarificación, ya que pueden existir diferentes significados del mismo (Fensham, 1985), lo que nos lleva otra vez a la cuestión de las finalidades de la educación científica, la idea de alfabetización científica y la concepción de la ciencia.

Como señalan Reid y Hodson (1989), los teóricos del currículo tienden a clasificar los diseños curriculares centrados en: a) la materia (estructura y métodos de la disciplina); b) el alumno (desarrollo de aspectos psicomotrices, cognitivos y afectivos); y c) la sociedad (inserción social y participación activa y responsable). A nuestro juicio, estas tres finalidades educativas deben estar presentes en todo proyecto curricular que pretenda ser equilibrado. Ahora bien, si se asume que cualquier propuesta fundamentada de la enseñanza de una materia -o un área de conocimientos- debe comenzar con una declaración de las finalidades que se pretenden con la misma, y que éstas no sólo derivan de la teoría del currículo sino también de la noción que se tenga de la materia o del área de conocimientos -la ciencia y la tecnología en el caso que nos ocupa-, parece claro que es necesario explicitar de alguna manera tales nociones y relacionarlas con las finalidades educativas de los currículos de ciencias y de tecnología.

\section{¿QUÉ NOCIONES DE CIENCIA Y TECNO- LOGIA PUEDEN SER UTILES PARA LA EDUCACION CIENTIFICA Y LA EDUCACIÓN TECNOLOGICA?}

Dar una definición de ciencia -o de tecnología- no es nada fácil porque, como se ha señalado en repetidas ocasiones, pueden significar muchas cosas. Sin embargo la cuestión es relevante, habiendo sido recogida por Aikenhead y otros (1989, véase también Aikenhead y Ryan, 1992) dentro de un apartado, en el que además se incluyen la comprensión del significado de investigación científica y desarrollo tecnológico (I+D) y de las relaciones entre ciencia y tecnología-las diferencias y las conexiones entre ambas-, del VOSTS (Views on Science-Technology-Society), instrumento elaborado para la evaluación de las creencias, concepciones y actitudes sobre numerosos aspectos del campo de conocimientos CTS.

En la actualidad, a la hora de definir, es frecuente poner más el acento en el proceso que conduce a la generación de resultados, es decir, en la práctica tecnológica y la práctica científica. Desde esta perspectiva, asumiremos en este trabajo el modelo conceptual de la práctica tecnológica de Pacey (1983), tal y como hemos hecho en diversas ocasiones (Acevedo, 1994, 1996 b, 1996 c), y la extensión del mismo que hemos realizado en otros lugares para la práctica científica (Acevedo, 1994, 1996 b).

En los cuadros 1 y 2 se muestran de forma esquemática, respectivamente, los modelos conceptuales de la práctica tecnológica y la práctica científica indicados. Cada uno de ellos abarca tres dimensiones: técnica, organizativa e ideológico-cultural. En ambos casos la dimensión técnica define lo que habitualmente se entiende, de manera restrictiva, por práctica tecnológica y práctica científica. La inclusión de las otras dos dimensiones, la organizativa y la ideológico-cultural, permite una generalización de los significados de tecnología y ciencia ampliados ahora con la entrada en consideración del ámbito social, que hemos denominado en cada modelo como sociotecnología y sociociencia. En el cuadro 3 se señalan algunos aspectos que sirven para describir las referidas dimensiones, tanto para la práctica tecnológica como para la práctica científica.

Pacey (1983) propone que el análisis, la valoración y la gestión de la tecnología se haga tomando como referencia las tres dimensiones en su conjunto, ya que los cambios en aspectos de cualquiera de ellas pueden producir ajustes y modificaciones en los de las otras dos. Además, mientras que las concepciones de la tecnología restringidas a la dimensión técnica tienden a proponer soluciones exclusivamente técnicas a los problemas tecnológicos de interés social, Pacey considera que muchas de las soluciones a los mismos dependen en mayor grado de cambios en los aspectos del ámbito sociotecnológico, esto es, de las dimensiones organizativa e ideológicocultural. Sin duda, esta otra forma de abordar los problemas tecnológicos que afectan a la sociedad puede favorecer la participación social para su resolución, por lo que es probable que las soluciones lleguen a estar más de acuerdo con los deseos e intereses de los ciudadanos.

Cuadro 1

Modelo conceptual de la tecnología según Pacey (1983).
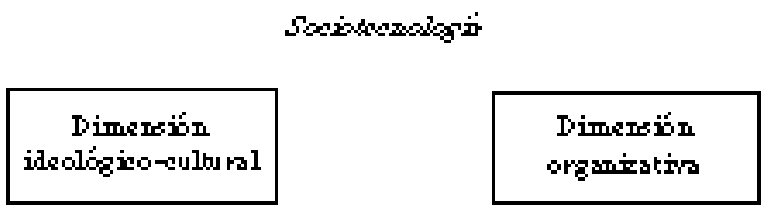

Práctica temógics

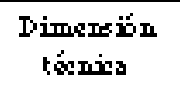

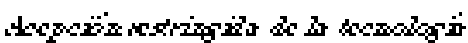


Cuadro 2

Modelo conceptual de la ciencia según Acevedo (1994).

\section{Sonimingir}

\begin{tabular}{|c|c|}
\hline $\begin{array}{c}\text { Dimensión } \\
\text { ideológico-sulbural }\end{array}$ & $\begin{array}{r}\text { Dimensión } \\
\text { orgmimativa }\end{array}$ \\
\hline
\end{tabular}

Prácties ciertífies

\section{Dimen×ín}

tónics

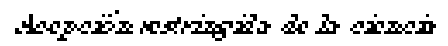

Basándose en el modelo de Pacey que acabamos de describir, Gilbert (1992) distingue, según qué aspectos se atiendan preferentemente en los contenidos -sean éstos factuales, conceptuales, procedimentales o actitudinales-, tres maneras de enfocar la educación tecnológica:

- Enseñanza para la tecnología, que se centra en los aspectos de la dimensión técnica del modelo de Pacey. Este enfoque está destinado sobre todo a dar a los estudiantes una formación de iniciación profesional con vista a su posible inserción en el mundo laboral y una orientación hacia los posibles estudios superiores ligados a la tecnología, de acuerdo con los intereses empresariales e industriales. Ésta es la perspectiva más habitual en la enseñanza, aunque también la más restringida.

- Enseñanza sobre la tecnología, que se dirige sobre todo a las cuestiones sociotecnológicas, es decir, a las relacionadas con las dimensiones organizativa e ideológico-cultural del modelo de Pacey. Esta orientación es característica de la educación CTS, especialmente en muchos cursos que se imparten dentro del ámbito de los estudios sociales y de humanidades; permite a los estu-

Dimensiones de la práctica tecnológica y la práctica científica.

\begin{tabular}{|c|c|}
\hline Práctica tecnológica & Práctica científica \\
\hline $\begin{array}{l}\text { Dimensión técnica } \\
\text { - Conocimientos disponibles. } \\
\text { - Capacidades y destrezas necesarias. } \\
\text { - Técnicas de fabricación y mantenimiento. } \\
\text { - Recursos humanos, instalaciones, etc. } \\
\text { - Herramientas, instrumentos, máquinas, etc. } \\
\text { - Materias primas, recursos físicos, productos obtenidos, de- } \\
\text { sechos y vertidos, etc. }\end{array}$ & $\begin{array}{l}\text { Dimensión técnica } \\
\text { - Conocimientos disponibles. } \\
\text { - Capacidades y destrezas necesarias. } \\
\text { - Métodos y procesos de investigación. } \\
\text { - Recursos humanos, laboratorios, etc. } \\
\text { - Instrumentación científica y tecnológica. } \\
\text { - Conocimientos producidos: ciencia privada y ciencia pública. }\end{array}$ \\
\hline $\begin{array}{l}\text { Dimensión organizativa } \\
\text { - Política tecnológica: planificación y gestión. } \\
\text { - Mercado, economía e industria. } \\
\text { - Sistema de recompensas en las comunidades de tecnólogos } \\
\text { (médicos, ingenieros, etc.). } \\
\text { - Sistema de relaciones entre agentes sociales (empresarios, } \\
\text { sindicatos, etc.). } \\
\text { - Actividad profesional productiva. } \\
\text { - Distribución de productos tecnológicos. } \\
\text { - Usuarios y consumidores de los productos tecnológicos. }\end{array}$ & $\begin{array}{l}\text { Dimensión organizativa } \\
\text { - Política científica: planificación y gestión. } \\
\text { - Fondos económicos y subvenciones a la investigación cien- } \\
\text { tífica. } \\
\text { - Sistema de recompensas en las comunidades de científicos } \\
\text { (físicos, biólogos, químicos, etc.). } \\
\text { - Relaciones entre grupos de investigación. } \\
\text { - Actividad profesional investigadora. } \\
\text { - Formas de difundir la ciencia: publicaciones, congresos, etc. } \\
\text { - Usuarios y consumidores de la ciencia. }\end{array}$ \\
\hline $\begin{array}{l}\text { Dimensión ideológico-cultural } \\
\text { - Finalidades y objetivos de la tecnología. } \\
\text { - Sistemas de valores y códigos éticos. } \\
\text { - Creencias sobre la técnica y el progreso. } \\
\text { - El papel de la creatividad en la tecnología. }\end{array}$ & $\begin{array}{l}\text { Dimensión ideológico-cultural } \\
\text { - Finalidades y objetivos de la ciencia. } \\
\text { - Sistemas de valores y códigos éticos. } \\
\text { - Creencias sobre la ciencia y el progreso. } \\
\text { - El papel de la creatividad en la ciencia. }\end{array}$ \\
\hline
\end{tabular}


diantes aprender algo sobre la influencia de los valores contextuales, la organización y la economía industrial, las consecuencias sociales de la tecnología, etc.

- Enseñanza en la tecnología, que toma en consideración todas las dimensiones del modelo de Pacey. Gilbert (1992) subraya que adoptar este punto de vista conduce a una enseñanza comprensiva y holística de la tecnología y a una educación tecnológica más equilibrada.

De manera similar, a partir del modelo que hemos propuesto para la práctica científica, también podemos diferenciar entre: a) enseñanza para la ciencia, basada en su dimensión técnica; $b$ ) enseñanza sobre la ciencia, orientada hacia los aspectos del ámbito sociocientífico que abarca a las dimensiones organizativa e ideológicocultural; y c) enseñanza en la ciencia, que pretende tomar en cuenta, de forma equilibrada, todas las dimensiones del modelo.

En nuestras aulas suele predominar, generalmente, el enfoque para la ciencia basado en la estructura de la disciplina, que casa mal con el principio de ciencia para todos, indicado anteriormente; pero en la actualidad parece estar empezando a darse un cierto desplazamiento en la educación científica hacia la enseñanza sobre la ciencia, de acuerdo con las ideas del movimiento CTS. Aunque nos parece muy importante asumir los aspectos que se tratan en tal enfoque, puede ser útil recordar aquí lo señalado por Reid y Hodson (1989) en relación con las modas de las reformas curriculares:

«Lo que resulta doloroso [...] es que los movimientos de reforma tiendan a perder la visión de otros énfasis y busquen sólo su orientación particular. Las cuestiones sociales, morales y económicas son importantes, pero no se deben olvidar las consideraciones centradas en la estructura de la disciplina. Necesitamos incluir tanto las cuestiones de valores como los productos y proceso de la ciencia, en lugar de sustituir unos por otros.» (p. 32 de la traducción española).

Por un lado, los modelos conceptuales de la práctica tecnológica y la práctica científica, cuando se toman en cuenta todas sus dimensiones tal y como se sostiene en las enseñanzas en la tecnología y en la ciencia, encajan bien con las finalidades educativas derivadas de la teoría del currículo que hemos señalado más arriba, sobre todo con las orientadas hacia la materia y la sociedad, y también pueden conectar con las centradas en el alumno, especialmente a través de la formación en valores y actitudes, lo que podría repercutir positivamente en la atención educativa en el campo afectivo. No obstante, los currículos de ciencias y de tecnología de hoy en día sin duda, están todavía algo lejos de alcanzar el deseado equilibrio propuesto por las enseñanzas en la ciencia o en la tecnología. Por otro lado, las nociones de práctica tecnológica y práctica científica que se desprenden, respectivamente, de dichos modelos pueden ser útiles, igualmente, para el análisis de criterios que permitan diferenciar-y también relacionar-entre ciencia y tecnología, lo que constituye el principal propósito de este trabajo.

\section{FUNDAMENTOS DE LA ELECCIÓN DE LOS CRITERIOS}

Con el fin de contribuir a aclarar la situación descrita, se analizarán algunos criterios destinados a intentar diferenciar entre ciencia y tecnología. Para fundamentar los mismos utilizaremos, además de las dimensiones de los modelos de la práctica científica y la práctica tecnológica expuestos en este trabajo, los denominados valores de la ciencia (Longino, 1983, 1990) y de la tecnología (Layton, 1988), distinguiendo, como hacen estos autores, entre valores constitutivos y valores contextuales.

Generalmente suele considerarse que los valores constitutivos son esenciales para la práctica científica. Éstos aparecen reflejados, por ejemplo, en el ethos normativo de la ciencia moderna establecido por Merton (1973), definido por el siguiente esquema: 1) Universalismo. El conocimiento científico debe mantenerse al margen de los prejuicios de tipo personal, de otra forma; tiene que ser independiente de otras razones distintas a las puramente científicas. 2) Comunalismo. El conocimiento científico debe considerarse una propiedad pública, tiene que evitarse el secreto y el hermetismo en su comunicación. 3) Desinterés. La ciencia debe cultivarse para hacer progresar el conocimiento en sí mismo y no por intereses particulares. 4) Escepticismo organizado. El conocimiento científico tiene que ser sometido a un examen crítico objetivo, debiendo considerarse provisional cualquier hipótesis no verificada por los métodos de la ciencia.

Estas normas constituyen uno de los campos de estudio característicos de la sociología de la ciencia mertoniana, que se centra sobre todo en los aspectos organizativos y los imperativos institucionales de la comunidad científica: los códigos éticos, el sistema de recompensas, las relaciones de roles y estatus, etc. Los valores constitutivos de la ciencia están ligados a las finalidades y objetivos de la práctica científica tal y como se perciben idealmente por las propias comunidades científicas; además, también suelen formularse como objetivos de la enseñanza de las ciencias.

En cambio, los valores contextuales de la ciencia se relacionan con el ambiente social, político y cultural en el que se desarrolla la práctica científica. Entre los mismos pueden citarse, como ejemplos, el utilitarismo, los beneficios económicos, las creencias religiosas, las ideologías políticas y la cuestión social del género en la ciencia. A menudo, se ha supuesto que la ciencia, la «buena ciencia» al menos, estaba al margen de estos valores, pero cada vez se están teniendo más en cuenta para comprender mejor la naturaleza de la ciencia y su práctica. La actividad científica es también un proceso social que incluye un conjunto de valores e intereses de la sociedad en la que está inmersa, los cuales están detrás de cada línea de investigación científica emprendida. Así ocurre, por ejemplo, cuando se consideran las áreas de conocimiento científico que reciben más apoyo social, moral y financiero por parte de los gobiernos y las empresas industriales. Sin embargo, los valores contex- 
tuales no han recibido todavía la atención que merecen en la enseñanza de las ciencias.

La cuestión de los valores, en la tecnología, ha sido algo menos tratada que en la ciencia (véase como excepción, p.e., Pacey, 1980, 1983) y, generalmente, la enseñanza de la tecnología se ha ocupado muy poco de ella. Es bastante probable que los tecnólogos tengan algunos valores propios similares a los de los científicos y otros investigadores. No obstante, cuando nos centramos en la organización de ambos tipos de comunidades, las diferencias entre los valores constitutivos de la ciencia y la tecnología pueden hacerse más evidentes. Desde luego, las normas ideales mertonianas de la ciencia académica no son las mismas que las de las comunidades de tecnólogos (Layton, 1988). Además, en el caso de la tecnología, la separación entre valores constitutivos y contextuales resulta más difícil y menos efectiva aún que en el de la ciencia, ya que allí es más fácil asumir la implicación de los valores contextuales por ser éstos también intrínsecos a la empresa tecnológica.

Resulta evidente, pues, que la práctica tecnológica tiene profundamente incorporada una serie de valores. Como acabamos de indicar, en la actividad tecnológica aparecen mezclados: $a$ ) valores constitutivos de la tecnología -tales como racionalidad técnica, «virtuosismo» tecnológico, eficiencia, estética, economía, etc.-; $b$ ) valores contextuales -como, por ejemplo, razones de beneficio económico, bienestar social, prestigio nacional o industrial, poder político, militar o empresarial, la influencia del género, etc.-; valores que subyacen en la elección de los problemas a resolver con la tecnología, en el propio diseño tecnológico y en los criterios que se utilizan para evaluar los resultados de la opción elegida. Por tanto, los dos tipos de valores se encuentran presentes en las innovaciones tecnológicas, suelen transmitirse además cuando se transfiere una tecnología - pudiendo en tal caso entrar en conflicto con los valores contextuales de la sociedad receptora- y se muestran en desacuerdo con los valores sociales dominantes cuando queda desfasada o se abandona una tecnología determinada.

Para diferenciar entre ciencia y tecnología hemos seleccionado tres criterios capaces de poner en juego tanto valores constitutivos como contextuales de ambas, así como diversos aspectos de las dimensiones de los modelos de la práctica tecnológica y la práctica científica asumidos en este trabajo. Estos criterios son:

a) las características propias del conocimiento tecnológico;

b) las actitudes ante las publicaciones: artículos versus patentes;

c) los propósitos de los laboratorios de investigación académica e industrial.

En cierto modo los dos últimos no son del todo independientes entre sí pero, por claridad en la exposición, los abordaremos por separado. Además, aceptamos de antemano que las profundas relaciones exis- tentes hoy en día entre la ciencia y la tecnología, dentro del complejo sistema conocido como tecnociencia, y los enormes cambios sociales producidos desde el nacimiento de la ciencia moderna, especialmente durante la segunda mitad del siglo XX, pueden dificultar la aplicación nítida de los mismos. De todas formas, los criterios elegidos permiten tratar cuestiones importantes de la actividad científica y tecnológica que habitualmente no se consideran en la enseñanza de las ciencias ni en la de la tecnología, pese a su interés para una mejor comprensión de la naturaleza y la práctica de ambas. Así mismo, muestran la influencia del contexto y en parte, como consecuencia de éste, las intrincadas y cambiantes relaciones entre ciencia y tecnología de la época actual respecto a las de otros momentos del pasado.

\section{CONOCIMIENTO TECNOLÓG ICO VERSUS CONOCIMIENTO CIENTÍFICO}

Gilbert (1995) ha establecido algunas diferencias entre el conocimiento científico y el tecnológico atendiendo a diversos aspectos: finalidad -explicación $v s$. fabricación-, interés -lo natural vs. lo artificial-, método -analítico vs. sintético-, procedimiento -simplificación del fenómeno vs. complejidad de la necesidad formula$\mathrm{da}-\mathrm{y}$ resultado -conocimiento generalizable $v s$. objeto particular-. Ahora bien, pese a que son sugerentes y estimulantes para el debate, en su artículo no prosigue con su desarrollo.

Hay que reconocer que, en la actualidad, la tecnología utiliza métodos sistemáticos de investigación semejantes a los de la ciencia; así mismo hace uso de los hallazgos de ésta. Sin embargo, esto no justifica la creencia de muchos profesores de ciencia, señalada por Rennie (1987), según la cual: «la tecnología se considera la aplicación con fines prácticos del conocimiento, las leyes y los principios científicos.» Estos profesores ven la tecnología como una forma de ciencia aplicada que está subordinada en gran medida a la ciencia básica. Ya hemos mostrado anteriormente (Acevedo 1994, 1996a) las deficiencias del modelo lineal y epistemológicamente jerárquico de las relaciones entre ciencia, tecnología y sociedad: «la tecnología no es sinónimo de ciencia aplicada». En efecto, la tecnología no se limita a tomar prestadas sus ideas de la ciencia para dar respuesta a determinadas necesidades humanas y a algunos problemas sociales importantes, sino que llega a configurar tales problemas (Acevedo, 1996c). Además, con tal fin, ha desarrollado sus propios métodos, perviviendo en ella algunas características específicas de los oficios tradicionales, conocimientos tácitos y habilidades técnicas (know-how), de acuerdo con el argot norteamericano al uso desde 1897 (Cardwell, 1994).

La creencia apuntada por Rennie (1987) ignora igualmente la posibilidad de teorías tecnológicas muy elaboradas. Para muchas personas, la existencia de teorías procedentes de la tecnología no es tan evidente como la 
de teorías científicas. No obstante, se han elaborado muchas, por ejemplo, desde la medicina, la informática o las diversas ingenierías; incluso algunas de ellas han contribuido, y continúan haciéndolo en buena medida, al desarrollo de las teorías científicas (Sanmartín, 1987, 1990). Ahora bien, conviene no olvidar, tal y como nos recuerda Mitcham (1989), que el criterio de validez de una teoría tecnológica no es tanto que sea «verdadera»o, en un plano más modesto, «verosímil» sino que funcione en la práctica y sea útil, lo que supone tener que diferenciar entre racionalidad científica y racionalidad tecnológica. En definitiva, aunque la ciencia es una fuente importante de conocimientos hay otras posibles en nuestra cultura occidental, siendo la tecnología una de ellas.

Por otra parte, el conocimiento tecnológico, que es esencialmente interdisciplinar y pragmático, está orientado hacia una praxis concreta para la resolución de problemas complejos y la toma de decisiones en cuestiones que afectan profundamente a la sociedad. En el mismo conviven conocimientos de carácter proposicional, obtenidos a partir de diversos campos, y conocimientos operacionales relacionados con el saber hacer. El conocimiento tecnológico tiene un carácter propio que lo diferencia, formal y sustancialmente, del originado por la ciencia básica. Así mismo, las operaciones de diseño, desarrollo y evaluación de tecnologías se ajustan a una lógica diferente y más compleja aún que la de la investigación científica.

Fleming (1989), siguiendo a Staudenmaier (1985), ha destacado los principales componentes que configuran el conocimiento tecnológico:

- Conceptos científicos que, en cualquier caso, tienen que ser elaborados de nuevo, rebajando su nivel de abstracción, para adaptarse a las necesidades y al contexto del proyecto de cada diseño tecnológico.

- Conocimiento problemático, referente a determinados aspectos discutibles de la actividad tecnológica, como pueden ser los posibles impactos sociales y medioambientales de una tecnología, ciertas dificultades que aparecen al adoptar una innovación tecnológica, las que surgen con una tecnología diseñada para un contexto determinado al transferirla a otros ambientes culturales diferentes, etc.

- Teoría tecnológica, considerada como un cuerpo de conocimientos que usa métodos experimentales sistemáticos similares a los de la ciencia pero centrados en el diseño, la construcción y el comportamiento de artefactos y sistemas tecnológicos. Una teoría tecnológica supone siempre una reflexión sobre la práctica tecnológica, por lo que puede considerarse como mediadora entre ésta y las teorías científicas más abstractas.

- Pericia técnica, o know-how, entendida como procedimientos y técnicas específicas con instrumentos y máquinas acompañados de un conjunto de criterios pragmáticos basados, sobre todo, en un conocimiento tácito casi imposible de codificar.
Es importante destacar aquí que estos componentes adquieren su pleno significado como consecuencia de la tensión existente entre el diseño tecnológico y las restricciones del contexto social y cultural (Staudenmaier, 1985); en otros términos, en el conocimiento tecnológico se ponen de manifiesto tanto los valores constitutivos como los contextuales de la tecnología.

Podemos concluir lo tratado para este criterio subrayando que, del mismo modo que existen comunidades de científicos, los ingenieros, médicos y otros profesionales forman comunidades de tecnólogos que tienen su propio ámbito de problemas, teorías, métodos, procedimientos y técnicas en donde se emplean elementos derivados del conocimiento científico, aunque adaptados a su peculiar y característico modo de hacer, conjuntamente con conocimientos tácitos de carácter técnico, tradiciones basadas en la experiencia acumulada y prácticas empresariales relacionadas con la comercialización y el uso de sus productos y servicios.

\section{ACTITUDES ANTE LAS PUBLICACIONES}

Según Price (1972) las actitudes de los científicos y los tecnólogos ante las publicaciones marcan una diferencia importante entre la ciencia y la tecnología. Las revistas especializadas de investigación científica contienen información avanzada de dominio público, y se supone que los científicos académicos elaboran artículos para dar a conocer formalmente los resultados de sus investigaciones a la comunidad científica a la que pertenecen. Sin embargo, como las relaciones más o menos informales entre los investigadores -favorecidas en los últimos años por la existencia de modernos y rápidos medios de comunicación como el fax, el correo electrónico o las redes informáticas- hacen que hoy en día la información más significativa fluya continuamente entre ellos, con frecuencia ésta es conocida antes de ser publicada, al menos por aquellos científicos que se encuentran en el frente de las líneas de investigación. Por lo tanto, quienes investigan en ciencia básica no publican solamente para cumplir con la norma, tácitamente admitida por la ciencia, de comunicar públicamente sus trabajos - norma expresada por el comunalismo mertoniano-, sino también, y quizás sobre todo, porque desean ver plasmados sus hallazgos en una revista científica prestigiosa, ya que ello, junto con las citas del artículo hechas por otros autores, suele implicar el reconocimiento institucional de su aportación a la ciencia (Guggenheim, 1982; Merton, 1973). Al mismo tiempo, los sistemas empleados para la promoción personal y la concesión de subvenciones a la investigación, dentro de la organización universitaria adoptada en muchos países, obligan todavía a la mayoría de estos científicos a publicar de manera imperiosa, a veces demasiado apresuradamente, siguiendo la conocida máxima de «publica o perece».

Ahora bien, hay que tener en cuenta que otros científicos trabajan en investigación industrial o tecnológica. En un trabajo clásico, Ellis (1972) mostró que muy pocos de éstos se oponen a las restricciones que se dan en las 
empresas para publicar artículos y que la mayoría comprenden y justifican esta situación. También señaló que estos científicos tampoco parecen estar demasiado preocupados por obtener su reputación mediante el sistema de publicaciones aún vigente fuera de la organización empresarial, ya que son otros sus intereses y motivaciones. En el caso de la tecnología, la tradición no es la misma que en la ciencia académica. El principal deseo de la mayoría de los tecnólogos y científicos industriales es contribuir a patentar en vez de publicar. Así mismo, hay que considerar que en las revistas técnicas los artículos no tienen generalmente la misma función que en el caso de las revistas científicas; sirven más bien para actualizar la información tecnológica y, sobre todo, para justificar el contenido más importante de tales revistas: los catálogos de productos y los anuncios publicitarios que muestran la situación de la técnica en cada tecnología. Price (1972) ha advertido también que esta diferencia introduce una dificultad añadida a la hora de escribir la historia de la tecnología de la misma manera que se escribe la historia de la ciencia.

Hecha esta distinción, conviene hacer referencia ahora a un fenómeno, relacionado con la sociología de las publicaciones, que es cada vez más habitual en la ciencia contemporánea. Aunque en líneas generales el progreso de las ciencias sigue apareciendo reflejado en las revistas de investigación científica y aún se continúa publicando a gran ritmo, está aumentando la tendencia a que parte del conocimiento producido no se difunda tan libremente como antes (Ferné, 1989), lo que está dando lugar a un nuevo marco de relaciones institucionales que entra en colisión con el tradicional comunalismo del esquema mertoniano de la ciencia académica y con la necesidad que tienen los científicos de comunicarse entre sí. En gran medida, esto es consecuencia de la creciente exigencia de interés tecnológico en la investigación científica, debido a la mayor dependencia de ésta de los encargos y subvenciones de los gobiernos y las empresas, así como de las nuevas formas organizativas derivadas de la creación de redes internacionales que controlan buena parte del conocimiento esencial y la difusión de ideas y resultados, sobre todo en algunos campos estratégicos de investigación punta. De esta manera, en el mundo actual, el consabido secreto relacionado con la organización de las investigaciones científicas de carácter industrial y militar se está extendiendo al campo de la ciencia académica, que cada vez está siendo más dirigida hacia finalidades tecnológicas.

El criterio analizado pone de manifiesto cómo los sistemas de recompensas de la ciencia académica y la tecnología -incluyendo aquí la ciencia industrial-, englobados en los aspectos de la dimensión organizativa de los modelos expuestos para la práctica científica y la práctica tecnológica, pueden influir mucho en las actitudes ante la comunicación pública del conocimiento -considerada ésta como un valor constitutivo de la ciencia académica-, y pueden establecer así una diferencia entre ésta y la tecnología.

En el caso de la ciencia moderna, desde su nacimiento en el siglo XVII, el interés por las publicaciones ha estado casi siempre de acuerdo con la norma del comunalismo mertoniano. Pero, por otra parte, los cambios sociales acaecidos desde entonces -sobre todo políticos y empresariales- están influyendo tanto en la ciencia académica actual que cada vez hay más restricciones y controles externos al dominio público del conocimiento científico, al menos en ciertas áreas de investigación (Acevedo 1997). Puede percibirse así cómo algunos valores contextuales -como el utilitarismo, los beneficios económicos, el prestigio nacional, los poderes político y militar, etc.- están condicionando, a través de diversos aspectos de las dimensiones del ámbito sociocientífico, ciertos valores considerados desde siempre como constitutivos de la ciencia académica. Debido a esto, se está empezando a cuestionar la utilidad general del criterio analizado por estar difuminándose en el presente algunas de las fronteras que antaño existían entre la ciencia y la tecnología.

\section{PROPÓSITOS DE LOS LABORATORIOS DE INVESTIGACIÓN}

Según Latour y Woolgar (1979), un laboratorio de investigación en ciencia básica puede verse, parcialmente al menos, como un centro de producción de artículos científicos destinados a su publicación en revistas de la especialidad. El dinero gastado en estos laboratorios parece emplearse fundamentalmente en elaborar conocimientos que a su vez generan artículos. Estos autores -sociólogos de la ciencia- describen el contenido de los mismos como un tipo de literatura persuasiva para convencer a sus lectores -los miembros de la correspondiente comunidad científica- de la validez de los resultados de las investigaciones realizadas en el laboratorio, para que de esta manera puedan pasar a formar parte de la ciencia pública de su época. Todo esto queda expuesto con nitidez en los siguientes párrafos extraídos de su libro:

«Dentro de los despachos [del laboratorio] los participantes [científicos] producen artículos comparando y contrastando esos diagramas con otros similares y con otros artículos de la bibliografía publicada.» (p. 63 de la edición española. Las anotaciones entre corchetes son nuestras).

«Los participantes reconocen que el principal objeto de su actividad es producir artículos.» (p. 85).

«El problema de los participantes era persuadir a los lectores de artículos [...] que debían aceptar como hechos sus enunciados.»(p. 85).

Así pues, la investigación científica que se realiza en los laboratorios parece tener como uno de sus fines la producción de conocimiento básico publicable destinado a aumentar la comprensión, explicación y predicción de eventos y fenómenos. Pero éste es tan sólo uno de sus propósitos. Hoy en día se persiguen tantas metas con la investigación científica que cualquier intento de describirla con brevedad resultaría parcial e insuficiente. 
En efecto, en los laboratorios científicos, la investigación que se realiza también puede estar orientada a la elaboración de conocimiento práctico encaminado a su aplicación tecnológica, incluso cuando tal conocimiento procede de la investigación básica universitaria, que a veces es estratégica o dirigida.

Pasando al campo industrial y tecnológico, el principal motivo que suele darse en las empresas para justificar la creación y financiación de laboratorios de investigación y desarrollo es que los avances en ciencia y tecnología que pueden conseguirse en los mismos conducen a la obtención de patentes, las cuales se convertirán en nuevos procesos de fabricación y productos comerciales mejores y quizás más baratos que los anteriores, lo que a su vez dará lugar a más beneficios económicos. Sin embargo, Basalla (1988) ha señalado que además de esta estrategia empresarial agresiva de la investigación industrial, que persigue las innovaciones tecnológicas, existe otra defensiva menos conocida. Un laboratorio industrial también puede estar destinado a generar patentes que probablemente nunca se convertirán en productos comerciales o en mejoras de los procesos de fabricación, sino que se utilizarán para protegerse frente a la amenaza potencial de otros competidores innovadores y defenderse así de las empresas rivales. De esta manera, puede mantenerse un laboratorio industrial con objetivos mucho más conservadores que los que se reconocen habitualmente, esto es, con el fin de moderar el ritmo de aparición de novedades tecnológicas en un determinado sector comercial y conservar así durante algún tiempo la hegemonía adquirida.

Por otro lado, tradicionalmente se ha considerado que la investigación académica suele disponer de mayor libertad para definir sus propios objetivos, mientras que las metas de la investigación industrial vienen siempre impuestas por los intereses comerciales de la empresa, tanto si responden a una estrategia agresiva como si lo hacen a una defensiva. Ahora bien, aunque en la actualidad todavía hay bastantes científicos que trabajan en las universidades con cierto grado de libertad en pos de la ciencia básica, no conviene olvidar que, cada vez más, también se hace investigación universitaria por encargo de las empresas y los gobiernos, sobre todo en los países más avanzados, de tal forma que buena parte de lo que se investiga en ciencia básica está siendo condicionado por finalidades y metas tecnológicas. En palabras de Pacey (1983):

«Gran parte de la ciencia opera de este modo, con objetivos situados fuera de la práctica tecnológica pero con una función práctica dentro de ella.» (p. 21 de la traducción española).

En relación con esta cuestión, acerca de quién debe marcar las finalidades y objetivos de la investigación científica, resulta necesario recordar aquí que uno de los elementos básicos de la política científica y tecnológica de cada nación es la asignación de recursos a los proyectos de I+D; hecho que se ha convertido en algo especialmente importante durante la segunda mitad del siglo $\mathrm{XX}$, debido tanto a la gran profesionalización de la ciencia contemporánea -y los cuantiosos gastos que origina-como al enorme interés social alcanzado por la ciencia y, muy especialmente, por la tecnología. Apoyando determinadas investigaciones y marginando otras, la sociedad y quienes la gobiernan influyen en la configuración de la ciencia y la tecnología que se hace en un país en una determinada época. Ahora bien, la necesidad social de planificar y gestionar los recursos destinados a financiar la investigación científica y el desarrollo tecnológico no debería conducir a los políticos y gestores a caer en un excesivo dirigismo, exclusivamente orientado hacia objetivos tecnológicos concretos a lograr a corto plazo, ya que la investigación básica, que prepara científicos y genera conocimientos, es también fundamental para la innovación tecnológica. En esta línea de opinión, Feinberg (1985) se muestra muy claro cuando dice que:

«Sería muy imprudente que la sociedad intentase dirigir la mayor parte de la investigación hacia objetivos tecnológicos específicos, porque no podemos predecir con exactitud y con gran antelación cuál será el enfoque de los problemas científicos no resueltos que nos conducirá a los resultados tecnológicos deseados. [...] Por desgracia, los científicos mismos a menudo prometen que de su investigación básica surgirán tecnologías específicas, quizá como manera de estimular la recepción de ayudas económicas. Tales promesas [...] muestran cortedad de miras.» (p. 274 de la traducción española).

Y todavía es más rotundo al manifestar que:

«Cuando se necesitan nuevos descubrimientos científicos para lograr determinada tecnología en particular, el mejor método para efectuar tales descubrimientos es la investigación corriente no dirigida. A veces dichos descubrimientos aparecen en áreas claramente relacionadas con la tecnología deseada y, a veces, en áreas aparentemente no relacionadas con ella.» (p. 275).

Si los propósitos de los laboratorios y de las investigaciones que en ellos se realizan están ligados de alguna manera a la publicación de artículos científicos y a la obtención de patentes industriales, resulta claro que parte de lo expuesto en el análisis de este criterio es complementario de lo desarrollado en el anterior. Como hemos mostrado, en ambos casos se ponen en juego valores constitutivos de la ciencia y la tecnología y la importante influencia de los valores contextuales, que alcanzan no sólo a qué se investiga sino incluso a la manera en que se construyen los conocimientos científicos y tecnológicos.

\section{IMPLICACIONES EDUCATIVAS DEL TEMA}

Algunas de las investigaciones realizadas en los últimos años sobre las concepciones CTS han puesto de manifiesto que muchos estudiantes y profesores tienen dificultades para distinguir entre ciencia y tecnología (véase un resumen de las mismas en Acevedo 1995, 1996c). Esta situación es común también a la de la mayoría de los 
ciudadanos, los cuales consideran que, de hecho, los éxitos científicos son triunfos tecnológicos y atribuyen a la ciencia buena parte de los problemas que origina la tecnología. Desde luego, hay que reconocer que, durante la segunda mitad del siglo XX, se han intensificando mucho las relaciones entre la ciencia y la tecnología y que estas relaciones están cambiando de orientación, especialmente si tenemos en cuenta la creciente imposición a la investigación científica de compatibilidad con las finalidades tecnológicas y que los criterios clásicos de racionalidad científica están empezando a ser desplazados por los de racionalidad tecnológica (Acevedo, 1997). La ciencia que, a finales del siglo XIX, se había apropiado jerárquica e interesadamente de la tecnología, se encuentra cada vez más al servicio de ésta, hasta el punto que un siglo después, en los albores del XXI, parece estar produciéndose un cambio del paradigma de investigación y desarrollo (I+D) por otro nuevo que es más desarrollo e investigación (D+I).

Los actuales currículos dirigidos a proporcionar una cultura científica y tecnológica para todos los ciudadanos y orientados por finalidades educativas centradas en el alumno, la ciencia, la tecnología y la sociedad, no pueden dejar al margen el análisis de las relaciones y diferencias entre la ciencia y la tecnología, tanto en el pasado como en el presente. En este sentido, no basta con reconocer que las actividades científicas conllevan diversas tecnologías y que para resolver problemas tecnológicos de hoy hacen falta ideas, conceptos y teorías científicas. Es preciso avanzar más en el significado de las nociones de ciencia y tecnología, incluyendo la presencia de lo social en la naturaleza y la práctica de las mismas, ya que ambas son construcciones humanas. Es necesario, pues, provocar la reflexión sobre los impactos que la ciencia y la tecnología ejercen en la sociedad, los cuales pueden alcanzar el sistema de valores sociales dominante, a veces incluso más allá de las finalidades y previsiones que inicialmente se pensaban. Al mismo tiempo, también hay que favorecer la comprensión de cómo los valores sociales intervienen como valores contextuales en la forma de desarrollarse, relacionarse y diferenciarse la ciencia y la tecnología.

A pesar de todo, muchos de los proyectos curriculares que intentan seguir estas orientaciones están contribuyendo también a confundir, en un sentido u otro, la ciencia y la tecnología. Así, por ejemplo, cuando se introducen algunos aspectos de la tecnología en la enseñanza de la ciencia, sea mediante el enfoque de ciencia integrada con tecnología o siguiendo cualquiera de las propuestas CTS que destacan, sobre todo las aplicaciones tecnológicas, es preciso hacer explícitas las características propias del conocimiento tecnológico que permiten diferenciarlo formal y sustancialmente del científico, de acuerdo con el criterio analizado. En caso contrario, como ya ha ocurrido en numerosas ocasiones (véanse algunos casos en Acevedo, 1995), es fácil llegar a considerar la tecnología como una consecuencia de la ciencia pura, jerárquicamente subordinada a ésta.
Por el contrario, los enfoques CTS que dan más énfasis a los aspectos sociales de la ciencia y la tecnología contemporáneas y a la influencia en éstas de los valores contextuales, centrándose casi exclusivamente en una enseñanza sobre ambas consideradas preferentemente como procesos sociales, suelen presentarlas como el complejo sistema global de nuestro tiempo llamado tecnociencia, lo que sin duda dificulta también poder diferenciarlas con alguna nitidez. Quizás esto es consecuencia de que ha sido probablemente en el ámbito social, en las dimensiones de la sociociencia y la sociotecnología de los modelos de la práctica científica y la práctica tecnológica que hemos tomado como referencia en este trabajo, donde se ha producido una mayor aproximación entre la ciencia y la tecnología, habiéndose difuminado más las fronteras entre las dos. Para evitar la confusión, en este caso consideramos necesario acudir a criterios como el de las actitudes ante las publicaciones y las patentes o el relacionado con las metas que se pretenden con los laboratorios de investigación académica e industrial, que, aunque en la actualidad parezcan estar perdiendo capacidad para diferenciar la ciencia de la tecnología, bien utilizados podrían favorecer una mejor comprensión de la evolución histórica de las relaciones y diferencias entre ambas, no sólo desde el punto de vista social sino incluso desde el epistemológico.

Nuestra conclusión es, por tanto, que la educación científica y la educación tecnológica, abordadas mediante una enseñanza integrada o por áreas separadas, deben contribuir a que los estudiantes lleguen a comprender algunas de las diferencias que se dan entre la ciencia y la tecnología, incluso para que también puedan entender mejor sus relaciones en el pasado, en el presente y las que se vislumbran para el futuro próximo. Determinados criterios que pueden utilizarse con tal fin, como los analizados en este trabajo, se centran en aspectos que no se tratan casi nunca en la enseñanza, pese a su interés para conocer cómo funcionan la ciencia y la tecnología. En esta línea, creemos que profesores y estudiantes deberían tener la posibilidad de visitar algún laboratorio de investigación de cada tipo y oportunidades para informarse ampliamente acerca de sus semejanzas y diferencias. Así mismo, tienen que ser conscientes de lo que suponen las publicaciones para los científicos, sobre todo si se considera el tiempo que éstos dedican a documentarse y a producir artículos, y de lo que significan las patentes en el cada vez más influyente mundo de la ciencia industrial y la tecnología.

\section{AGRADECIMIENTOS}

La versión final de este trabajo se ha visto notablemente enriquecida gracias, en buena medida, a las sugerencias y críticas de las dos personas que evaluaron y asesoraron el primer envío. 


\section{REFERENCIAS BIBLIOGRÁFICAS}

ACEVEDO, J.A. (1994). La dimensión social de la ciencia y la tecnología. Una perspectiva CTS del desarrollo tecnológico y su evaluación. Ponencia expuesta en las Jornadas de Industrias Químicas: En torno a treinta años de historia. AIQB y CEP, Huelva.

ACEVEDO, J.A. (1995). Educación tecnológica desde una perspectiva CTS. Una breve revisión del tema. Alambique, 3 , pp. 75-84.

ACEVEDO, J.A. (1996a). Cómo puede contribuir la historia de la técnica y la tecnología a la educación CTS. Resúmenes de los XVII Encuentros de Didáctica de las Ciencias Experimentales, pp. 119-120. La Rábida (Huelva).

ACEVEDO, J.A. (1996b). La educación CTS en el bachillerato LOGSE: la materia optativa ciencia, técnica y sociedad en Andalucía. Resúmenes de los XVII Encuentros de Didáctica de las Ciencias Experimentales, pp. 134-135. La Rábida (Huelva).

ACEVEDO, J.A. (1996c). La tecnología en las relaciones CTS. Una aproximación al tema. Enseñanza de las Ciencias, 14(1), pp. 35-44.

ACEVEDO, J.A. (1997). ¿Publicar o patentar? Hacia una ciencia cada vez más ligada a la tecnología. Revista Española de Física, 11(2), pp. 8-11.

AIKENHEAD, G.S., RYAN, A.G. y FLEMING, R.W. (1989). Views on science-technology-society (form CDN. mc. 5). Department of Curriculum Studies, University of Saskatchewan, Saskatoon, Canadá, S7N 0W0.

AIKENHEAD, G.S. y RYAN, A.G. (1992). The development of a new instrument: Views on Science-Technology-Society (VOSTS). Science Education, 76(5), pp. 477-491.

ALLSOP, R.T. y WOOLNOUGH, B.E. (1990). The relationship of technology to science in English schools. Journal of Curriculum Studies, 22(2), pp. 127-136.

BASALLA, G. (1988). The evolution of technology. Cambridge University Press, Cambridge. Trad. de Vigil, J., 1991. La evolución de la tecnología. Barcelona: Crítica.

BERNAL, J.D. (1964). Science in History. Watts, Londres. Trad. de Capella, J.R., 1967. Historia social de la ciencia. Barcelona: Península.

CARDWELL, D. (1994). The Fontana History of Technology. Harper Collins Publishers, Londres. Trad. cast., 1996. Historia de la tecnología. Madrid: Alianza.

CASALDERREY, M.L. (1986). Aproximación a la integración ciencia-tecnología. Madrid: Servicio de Publicaciones del MEC.

DES (1977). Curriculum, 11-16. Londres: HMSO.

DES (1985). Science, 5-16. Londres: HMSO.

ELLIS, N.D. (1972). The occupation of science, en Barnes, R. (ed.), Sociology of science. Penguin Books, Harmondsworth, 188-205. Trad. de Míguez, N.A., 1980. Estudios sobre sociología de la ciencia, pp. 185-202. Madrid: Alianza.

FEINBERG, G. (1985). Solid clues. Simon \& Schuster, Nueva York. Trad. de Iglesias, J., 1986. Claves ciertas. Barcelona: Salvat.

FENSHAM, P.J. (1985). Science for all: A reflective essay. Journal of Curriculum Studies, 17(4), pp. 415-435.
FERNÁNDEZ-URÍA, E. (1979). Estructura y didáctica de las Ciencias. Madrid: Servicio de Publicaciones del MEC.

FERNÉ, G. (1989). La ciencia, una nueva mercancía. Mundo científico, 9(91), pp. 564-571.

FLEMING, R.W. (1987). High-school graduates' beliefs about science-technology-society II. The interaction among science, technology and society. Science Education, 71(2), pp. 163-186.

FLEMING, R.W. (1989). Literacy for a technological age. Science Education, 73(4), pp. 391-404.

GILBERT, J.K. (1992). The interface between science education and technology education. International Journal of Science Education, 14(5), pp. 563-578.

GILBERT, J.K. (1995). Educación tecnológica: una nueva asignatura en todo el mundo. Enseñanza de las Ciencias, 13(1), pp. 15-24.

GUGGENHEIM, A. (1982). Cuando los sociólogos estudian la literatura científica. Mundo científico, 2(20), pp. 1224-1226.

LATOUR, B. y WOOLGAR, S. (1979). Laboratory Life: The Social Construction of Scientific Facts. Sage, Londres. (2a. edición, 1986, Princenton: Princenton University Press). Trad. de Pérez Sedeño, E., 1995. La vida en el laboratorio. La construcción de los hechos científicos. Madrid: Alianza.

LAYTON, D. (1988). Revaluing the T in STS. International Journal of Science Education, 10(4), pp. 367-378.

LONGINO, H.E. (1983). Beyond Bad Science: skeptical reflections on the value-freedom of scientific inquiry. Science, Technology and Human Values, 8(1), pp. 7-17.

LONGINO, H.E. (1990). Science as social knowledge: Values and objectivity in scientific inquiry. Princenton: Princenton University Press.

MEC (1991). Real Decreto 1007/1991, de 14 de junio, por el que se establecen las enseñanzas mínimas correspondientes a la Educación Secundaria Obligatoria. Madrid: BOE.

MEDWAY, P. (1989). Issues in the theory and practice of technology education. Studies in Science Education, 16, pp. 1-24.

MERTON, R.K. (1973). The sociology of science. Theoretical and empirical investigations. Chicago: University of Chicago Press. Trad. de Míguez, N.A., 1977. La sociología de la ciencia. Investigaciones teóricas yempíricas. Madrid: Alianza.

MITCHAM, C. (1989). ¿Qué es la filosofía de la tecnología? Barcelona: Anthropos.

PACEY, A. (1980). El laberinto del ingenio. Ideas e idealismo en el desarrollo de la tecnología. Barcelona: Gustavo Gili. Traducción del original en inglés (1974).

PACEY, A. (1983). The Culture of Technology. Cambridge: Cambridge MIT Press. Trad. de Ríos, R., 1990. La cultura de la tecnología. México DF: FCE.

PRICE, D.J. DE SOLLA (1972). Science and technology: Distinctions and interrelationships, en Barnes, R. (ed.): Sociology of science. Harmondsworth: Penguin Books, pp. 166-180. Trad. de Míguez, N.A., 1980. Estudios sobre sociología de la ciencia, pp. 163-177. Madrid: Alianza.

PRICE, R.F. y CROSS, R.T. (1995). Conceptions of science and technology clarified: improving the teaching of science. 
International Journal of Science Education, 17(3), pp. 285-293.

REID, D.J. y HODSON, D. (1989). Science for all. Cassell, Londres. Trad. de Martín-Díaz, M.J. y García-Lucía, L.A., 1993. Ciencia para todos en secundaria. Madrid: Narcea.

RENNIE, L.J. (1987). Teachers' and pupils' perceptions of technology and the implications for curriculum. Research in Science \& Technological Education, 5(2), pp. 121-133.

SANMARTÍN, J. (1987). Los nuevos redentores. Reflexiones sobre la ingeniería genética, la sociobiología y el mundo feliz que nos prometen. Barcelona: Anthropos.
SANMARTÍN, J. (1990). Tecnología y futuro humano. Barcelona: Anthropos.

STAUDENMAIER, J.M. (1985). Technology's Storytellers: Reweaving the Human Fabric. Cambridge: MIT Press.

UNESCO (1990). The teaching of science and technology in an interdisciplinary context. Science and Technology Education Document Series, 38. París: UNESCO.

WOOLNOUGH, B.E. (1988). Technological Education and Science in Schools. ASE, Hatfield, Herts.

[Artículo recibido en febrero de 1997 y aceptado en octubre de 1997.] 